\title{
Emerging Roles of NPQ/Spexin in Physiology and Pathology
}

\author{
Shuang-Yu Lv', Yu-Chen Zhou', Xiao-Mei Zhang ${ }^{1}$, Wei-Dong Chen ${ }^{1,2 *}$ and \\ Yan-Dong Wang ${ }^{3 *}$
}

${ }^{1}$ Key Laboratory of Receptors-Mediated Gene Regulation and Drug Discovery, School of Medicine, Henan University, Kaifeng, China, ${ }^{2}$ Key Laboratory of Molecular Pathology, School of Basic Medical Science, Inner Mongolia Medical University, Hohhot, China, ${ }^{3}$ State Key Laboratory of Chemical Resource Engineering, College of Life Science and Technology, Beijing University of Chemical Technology, Beijing, China

OPEN ACCESS

Edited by:

Andres Trostchansky,

University of the Republic, Uruguay

Reviewed by:

Lila Oyama,

Federal University of São Paulo, Brazil

Anderson O. L. Wong,

The University of Hong Kong,

Hong Kong

*Correspondence:

Wei-Dong Chen

wdchen666@163.com

Yan-Dong Wang

ydwangbuct2009@163.com

Specialty section:

This article was submitted to

Experimental Pharmacology and

Drug Discovery,

a section of the journal

Frontiers in Pharmacology

Received: 26 December 2018

Accepted: 11 April 2019

Published: 07 May 2019

Citation:

Lv S-Y, Zhou Y-C, Zhang X-M,

Chen W-D and Wang Y-D (2019)

Emerging Roles of NPQ/Spexin in

Physiology and Pathology.

Front. Pharmacol. 10:457.

doi: 10.3389/fphar.2019.00457
Spexin (SPX), also called neuropeptide Q (NPQ), is a novel endogenous neuropeptide. Spexin gene and protein are widely expressed in central nervous system and peripheral tissues in humans, rodents, goldfish, etc. A few of physiological and pathological roles of spexin are gradually emerged recently. This article summarized the roles of spexin in feeding behavior, gastrointestinal motility, obesity, diabetes, energy metabolism, endocrine, mental diseases, and cardiovascular function. Given the broad roles of spexin, this neuropeptide has attracted much interest from investigators and will be as a promising future target for novel therapeutic research and drug design.

Keywords: spexin, NPQ, obesity, metabolism, neuropeptide

\section{INTRODUCTION}

Spexin (SPX), namely neuropeptide Q (NPQ), is a new identified peptide hormone. Spexin was first identified in human genome through bioinformatic method based on a hidden Markov model (Mirabeau et al., 2007). Then spexin was confirmed by biochemical method and was first detected in the esophagus and stomach of mice (Mirabeau et al., 2007). The prepropeptide human spexin containing 116 amino acid residues was encoded by C12ORF39 gene (Wan et al., 2010). In humans, the precursor of spexin contains a signal peptide, two prohormone cleavage sites, and predicted processed peptide (Sonmez et al., 2009). The small amino acid sequence between dibasic cleavage sites with C-terminal amidation was called spexin (Sonmez et al., 2009). The nuclear magnetic resonance (NMR) analysis indicates that the molecular surface of spexin (gold fish) is largely hydrophobic except for Lys ${ }^{11}$, and its 3D structure is an a-helix from $\mathrm{Gln}^{5}$ to $\mathrm{Gln}^{14}$ with a flexible $\mathrm{NH}_{2}$ terminus from $\mathrm{Asn}^{1}$ to $\mathrm{Pro}^{4}$ (Wong et al., 2013). The amino acid sequence of the spexin is highly conserved among various species (Figure 1; Wong et al., 2013), suggesting that the peptide has an essential property during biological evolution and may be potentially involved in regulating a variety of physiological and pathological functions. The spexin paralogous gene (spexin2) was identified in vertebrate chromosomes. However, mammals do not have spexin2, like spexin (namely spexin1). Spexin2 encodes a mature peptide containing 14 amino acids with amidation at the $C$ terminus, named spexin2 (Figure 1; Kim et al., 2014).

Spexin was proved to be a natural ligand for galanin receptor 2/3 (GALR2/3) (Kim et al., 2014). The receptor-ligand binding assay demonstrates that it activated humans, Xenopus, and zebrafish GALR2/3 family receptors but not GALR1 (Kim et al., 2014). Reyes-Alcaraz et al. found that the spexin and galanin induce specific active conformations of GALR2, indicating 


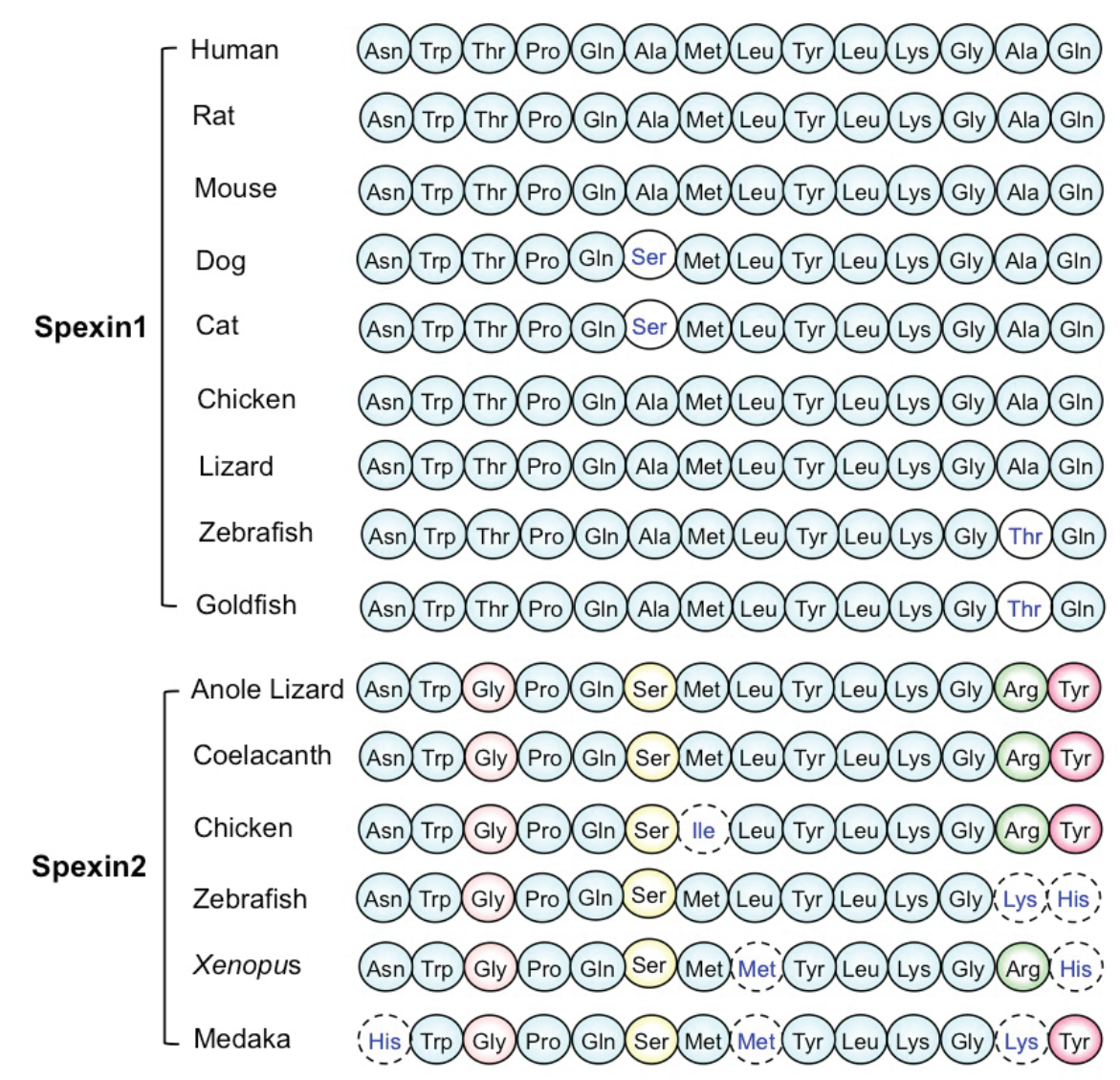

FIGURE 1 | The amino acid sequence of mature spexin1 and spexin2 in various species. (The figure modified from Wong et al., 2013; Kim et al., 2014).

that the two ligands activate differential signaling via the same receptor, GALR2 (Reyes-Alcaraz et al., 2018). Galanin, as a classic neuropeptide, has a regulation on numerous physiologic and pathophysiological processes, including feeding and energy homeostasis, osmotic regulation and water intake, pain, regeneration and neurite outgrowth, Alzheimer's disease, cerebral ischemia and stroke, seizures and epilepsy, anxiety disorders, depression, etc. (Lang et al., 2015).

Spexin mRNA and protein are widely distributed in central nervous system (CNS) and peripheral tissues in various species. In humans, spexin mRNA and spexin cytoplasmic immunoreactivity were found in skin, lung, stomach, small intestine, colon, liver, pancreatic islets, thyroid, adrenal gland, visceral fat, kidney, etc. ( $\mathrm{Gu}$ et al., 2015). In rats, high levels of mRNA were detected in brain, hypothalamus, esophagus, liver, kidney, thyroid, and ovary (Porzionato et al., 2010). The rat spexin-like immunoreactivity was strongly determined in epidermis and sebaceous glands of skin, epithelium of esophagus, base of glands in stomach, epithelium of small intestine, hepatocytes, etc. (Porzionato et al., 2010). In goldfish, spexin mRNA was confirmed in CNS and peripheral organs, especially in the optic tectum, hypothalamus, brain stem, liver, and testis (Wong et al., 2013). The broad distribution of spexin suggests multiple physiological and pathological functions of spexin.

\section{FEEDING AND ENERGY METABOLISM}

In Ya-fish, the spexin mRNA level in forebrain of postprandial group was higher than preprandial group (Wu et al., 2015). The mRNA expression of spexin in forebrain was reduced in the fasting group comparing with the feeding group, while the spexin mRNA was elevated after refeeding at the 7th day comparing with the feeding group. The spexin mRNA in forebrain was determined by different feeding status in Ya-fish, suggesting that spexin gene expression was controlled by different feeding conditions or metabolic status (Wu et al., 2015). Deng et al. have cloned and analyzed spexin gene from spotted scat (Scatophagus argus). And it was reported that food deprivation upregulated the spexin mRNA level in hypothalamus of spotted scat; however, the spexin mRNA level was decreased after refeeding for the unfeeding group, compared with the still unfeeding (fasting) group (Deng et al., 2018). In flatfish, the spexin mRNA in the hypothalamus was upregulated under the fasted condition (Wang et al., 2018). Wu et al. found that the contents of insulin and spexin in plasma and the mRNA levels of insulin and spexin in liver were all increased after refeeding in the 3-day-fasted goldfish, comparing with unfeeding group (Ma et al., 2017). Intraperitoneal (i.p.) and intracerebroventricular (i.c.v.) injection of spexin reduced food intake during a 2 -h feeding period in 
goldfish (Wong et al., 2013). And i.c.v. spexin inhibited the increase of food intake induced by neuropeptide (NPY) and orexin treatment. The inhibitory effect of central spexin on goldfish feeding behavior was induced by downregulation of orexigenic factors, including NPY, agouti gene-related protein (AgRP) and apelin, and upregulation of anorexigenic factors, including cholecystokinin (CCK), proopiomelanocortin (POMC), amphetamine-regulated transcript (CART), melanin-concentrating hormone $(\mathrm{MCH})$, and corticotropin-releasing hormone $(\mathrm{CRH}$; Wong et al., 2013). In orange-spotted grouper, spexin mRNA in hypothalamus was upregulated by 7 days of food deprivation, whereas the spexin gene was downregulated after refeeding treatment, compared with the group fasted for 7 days ( $\mathrm{Li}$ et al., 2016). Peripheral injection of spexin stimulated pomc mRNA expression and inhibited orexin mRNA expression in hypothalamus of the orange-spotted grouper ( $\mathrm{Li}$ et al., 2016). Zheng et al. found that spx1 knockout Zebrafish exhibited a higher food intake than the wild type (WT; Zheng et al., 2017). The agoutirelated protein 1 (agrp1) gene expression was higher in $s p x 1^{-/-}$ mutant fish than in WT fish after feeding, and intracranial treatment with spexin 1 decreased the agrpl gene expression in the zebrafish hypothalamus (Zheng et al., 2017). This result indicates that the inhibitory effect of spexin1 on food intake might be medicated by agrp1 gene (Zheng et al., 2017). In fish, under the fasting condition, the spexin gene was decreased in the forebrain of Ya-fish, whereas it was increased in the hypothalamus of spotted scat, flatfish, and orange-spotted grouper, comparing with the fed control. And the increased/decreased spexin gene expression of the fasting group was recovered when the food was available again (refeeding). Central or peripheral administration of spexin reduced food intake in goldfish.

In diet-induced obesity (DIO) rats, chronic subcutaneous (s.c.) injection with spexin reduced food intake (Walewski et al., 2014). These results indicate a solid inhibitory effect of exogenous spexin on food intake as well as a closed relationship between spexin and food control.

It is well established that the long-chain fatty acid (LCFA) uptake and storage are a key factor for body weight control (Petrescu et al., 2005). Spexin treatment produced a suppression effect on LCFA uptake into adipocytes isolated from untreated DIO mice (Walewski et al., 2014). Chronic i.p. injection of spexin reduced the respiratory exchange ratio (RER) at night and increased locomotor activity in DIO mice (Walewski et al., 2014). These results could explain the inhibitory effect of spexin on the body weight of mice with obesity. Recent report shows that chronic i.p. injection with spexin diminished hepatic lipids, serum alanine aminotransferase (ALT), and aspartate aminotransferase (AST) in mice with hepatic steatosis/ nonalcoholic fatty liver disease (HS/NAFLD; Jasmine et al., 2016). In hepatocytes isolated from DIO mice, spexin treatment reduced LCFA uptake (Jasmine et al., 2016). These results demonstrate a potentially beneficial effect of spexin in NAFLD treatment. In vitro, the high level of insulin induced by glucose stimulated spexin gene expression in both goldfish hepatocytes and brain cells (Ma et al., 2017). Human study shows that the serum spexin was negatively correlated with age, BMI, fasting glucose, and TG in healthy adult women, suggesting that spexin could independently predict the risk of high BMI and high fasting glucose (Lin et al., 2018a). In human adipocytes and murine 3 T3-L1 cells, spexin exhibited a stimulatory effect on lipolysis and an inhibitory effect on lipogenesis and glucose uptake (Kolodziejski et al., 2018). Clinical study shows that the serum spexin had a modest association with components of metabolic syndrome (MetS) only in women (Al-Daghri et al., 2018b). The suppression of spexin on lipogenesis, LCFA uptake, and RER and the promotion of spexin on lipolysis and motor activity indicate that spexin is a key negative regulator for energy budget.

\section{OBESITY AND DIABETES}

Spexin is a potentially regulating factor in obesity and energy metabolism (Wang et al., 2016). After daily injection (i.p.) with spexin for 6 days, the body weight of the high-fat-diet mice was reduced (Walewski and Berk, 2013). Clinical study shows that the spexin gene and protein were downregulated in omental and subcutaneous fat in obese patients compared with the normal fat (Walewski and Berk, 2013; Walewski et al., 2014). The concentration of serum spexin in obese patients was lower than that of the healthy participants (Walewski and Berk, 2013). Moreover, the serum spexin was markedly negative correlation with leptin in the obese patients (Walewski and Berk, 2013; Walewski et al., 2014). In children, the circulating serum spexin level in obese people was lower than that of the normal weight person (Kumar et al., 2016; Chen et al., 2019), indicating a potential role of spexin in childhood obesity. The circulating spexin level was inversely correlated with leptin in adolescents with obesity (Kumar et al., 2017). The increasing energy expenditure of spexin was in accordance with the decreasing body weight of spexin. However, the underlying mechanism needs to be further explored. More research is wanted to develop a spexin-based antiobesity drug.

In human tissues, the spexin immunoreactive cells and spexin mRNA were detected in endocrine and epithelial tissues, such as adrenal gland, visceral fat, thyroid, liver, etc. (Gu et al., 2015). The results indicate a potential role of spexin in modulating metabolism. Karaca et al. found that patients with type 1 diabetes mellitus (T1DM) have a lower level of spexin, not related to body mass index (BMI), glucose, or lipid parameters, comparing with healthy subjects (Karaca et al., 2018). Serum spexin levels in type 2 diabetes mellitus (T2DM) were lower than that of healthy subjects, and spexin was negatively correlated with blood glucose, triglyceride (TG), hemoglobin A1c (HbA1c), and low-density lipoprotein-cholesterol (LDL-C; Gu et al., 2015, 2016). However, Hodges et al. found that the serum spexin concentration did not show difference between the three groups (normal weight, obese, and obese with T2DM) in adolescents (Hodges et al., 2018). Recently, it was shown that the level of spexin exhibited a notably increase after 6 months in the pregnant women with gestational diabetes mellitus (GDM; Al-Daghri et al., 2019).

In adolescent patients with obesity or T2MD, the circulating spexin level is inversely correlated with blood glucose, leptin, 
lipids, etc. However, in adult patients with obesity or T2MD, the circulating spexin level does not exhibit a correlation with blood glucose, leptin, triglyceride (TG), etc. (Hodges et al., 2018). These results indicate that spexin exerts different pathological effects on obesity and metabolism between adults and adolescents. Al-Daghri et al. show that the level of spexin in serum had a modestly relationship with glucose and insulin sensitivity in pregnant women but had no influence on GDM and obesity (Al-Daghri et al., 2018a). In obese children, spexin was negatively correlated with insulin resistance and pancreatic $\beta$-cell function indicators (Chen et al., 2019). Recently, Sassek et al. have shown that spexin not only inhibited the insulin secretion from cultured cells and isolated islets induced by glucose in vitro but also reduced the insulin secretion in obese rats in vivo (Sassek et al., 2018a). Sassek et al. found that spexin was located inside the $\beta$ cells of the pig pancreas, and the release of spexin from islets was increased after a short term and decreased after a long term following treatment with glucose (Sassek et al., 2018b). In addition, spexin could improve the cell viability and proliferation of pancreatic islet cells and upregulate the protein level of proliferating cell nuclear antigen (PCNA; Sassek et al., 2018a).

\section{GASTROINTESTINAL MOTILITY AND BILE ACID}

The spexin mRNA was located in the submucosal layer of esophagus and stomach fundus of mouse using hybridization in situ (Mirabeau et al., 2007). The rat stomach explant assay indicates that spexin caused a contractile activity of stomach muscle (Mirabeau et al., 2007). The result indicates a potential role of spexin in regulation of digestive tract function. Spexin (i.p.) increased the mouse intestinal transit rate and promoted the colonic bead expulsion in vivo, and spexin obviously stimulates the intestinal/colonic muscular contractions in vitro (Lin et al., 2015). Spexin exerts the stimulatory effect on bowel motility through GALR2 receptor by activating L-type voltagedependent calcium channel (Lin et al., 2015). The findings demonstrate that spexin is an important regulator in gastrointestinal function. However, whether the central spexin has a similar effect on gastrointestinal motility and whether the effect of spexin on bowel motility via CNS needs to be identified in the future study.

Current study shows that spexin was involved in bile acid synthesis (Lin et al., 2018b). Peripheral (i.p.) treatment with spexin decreased the total bile acid level and cholesterol 7a-hydroxylase 1 (CYP7A1) mRNA level in the mouse liver, and this effect could be blocked by the GALR2 and GALR3 antagonists (Lin et al., 2018b).

\section{ENDOCRINE REGULATION}

Recent report shows that spexin may be a neuroendocrine signal related to multiple functions (Ma et al., 2018). In zebrafish, spexin mRNA was highly expressed in brain and ovary.
Incubated with spexin obviously inhibits release of luteinizing hormone (LH) in cultured goldfish pituitary cells, and i.p. injection with spexin reduced goldfish serum LH levels (Liu et al., 2013). The spexin gene expression in hypothalamus was affected by gonadal hormones in female goldfishes (Liu et al., 2013), implicating a potential role of spexin in reproduction and endocrine. Trudeau proposed that spexin, as an inhibitory neuropeptide, regulates LH in teleosts (Trudeau, 2018). Wang et al. have cloned the spexin gene from a flatfish, the halfsmooth tongue sole (Cynoglossus semilaevis; Wang et al., 2018). Treatment (i.p) with spexin upregulated the gnih (gonadotropin inhibitory hormone) and gnrh3 (gonadotropin-releasing hormone 3 ) gene expression and downregulated the $g h$ (growth hormone), $f \operatorname{sh} \beta$ (follicle-stimulating hormone $\beta$ ), and gth $\alpha$ (gonadotropin hormone $\alpha$ ) gene expression in the hypothalamus of the halfsmooth tongue sole (Wang et al., 2018). Treatment with $17 \beta$-estradiol (E2) decreased the spexin gene expression in the hypothalamus of the spotted scat in vitro and in vivo (Deng et al., 2018). These results suggest that spexin played a role in regulating reproductive function, especially inhibiting the LH secretion. However, all the present research has only utilized fish model. The animal models of mammalian and clinical studies are needed in the future.

An in vitro experiment shows that the incubation with spexin increased aldosterone secretion in rat isolated zona glomerulosa $(\mathrm{ZG})$ and stimulated corticosterone secretion in rat adrenocortical primary cells (Rucinski et al., 2010), indicating that spexin was involved in controlling the adrenocortical secretory function. In addition, BrdU assay shows that spexin inhibited adrenocortical cell proliferation (Rucinski et al., 2010).

\section{PAIN, ANXIETY, AND DEPRESSION}

Currently, spexin was reported to play a role in pain, anxiety, and depression. I.c.v. administration of spexin produced antinociceptive effect in the mouse warm water tail withdrawal assay (Toll et al., 2012). Female rats injected with spexin in hippocampal CA1 show a decrease in pain sensitivity in the formalin test, indicating an analgesic effect on tonic pain (Pirzeh and Taherianfard, 2014). Intra hippocampal CA3 (IHCA3) administration of spexin inhibited the pain sensitivity in the both early and late phrases of the rat formalin test (Moazen et al., 2017). Lv et al. found that centrally administrated spexin produced antinociceptive effect against inflammatory pain by dynorphin/א-opioid receptor pathway (Lv et al., 2019). All of the results indicate the analgesic effect of central spexin; however, the effect of spexin at the peripheral level is not clear. A comparison between spexin and morphine in antinociceptive efficiency needs to be evaluated. The synergistic analgesic relationship of morphine and spexin is still unknown.

Reyes-Alcaraz et al. demonstrate that i.c.v. injection with spexin-based GALR2-specific agonists induced an anxiolytic effect in mice (Reyes-Alcaraz et al., 2016). Chronic i.p. injection of SSRI antidepressant escitalopram caused a decrease in spexin gene expression in hypothalamus and an increase in spexin gene expression in the hippocampus and striatum in rats 
(Palasz et al., 2016). This study suggests that spexin was involved in regulating the depression. Palasz et al. found that chronic i.p. administration with haloperidol and chlorpromazine, the classical antipsychotic drugs, increased the spexin and pomc mRNA levels while decreased the kisspeptin-1 mRNA level in the rat amygdala (Palasz et al., 2018). It indicates that the neuropeptide spexin in the amygdala may be related to the mechanism of the antipsychotic drugs.

\section{OTHER ROLES}

Rats injected (i.c.v.) with spexin exhibited an increase in mean arterial pressure, a decrease in heart rate, and a marked decrease in urine flow rate (Toll et al., 2012), indicating that central spexin was involved in modulating the cardiovascular and renal activities. Spexin mRNA levels of carotid body in hyperoxiaexposed rats were higher than normoxia-exposed group, suggesting spexin in carotid body may be a regulator in hyperoxia-induced plasticity (Porzionato et al., 2012).

\section{CONCLUSION/PERSPECTIVES}

In summary, spexin, a novel biologically active peptide, has a broad distribution in CNS and periphery and is involved in regulating multiple physiological functions (Figure 2). Spexin inhibits food intake and body weight, promotes gastrointestinal motility, and exhibits a negative correlation with leptin in the obese patients. Serum spexin levels in T2DM are decreased and negatively correlated with blood glucose, TG, etc. The human studies are needed to be determined the potential of therapeutic strategies targeting spexin on T2DM. Spexin was shown to suppress LCFA uptake into adipocytes and alleviate hepatic injury of mice with HS/NAFLD. In addition, spexin inhibits release of $\mathrm{LH}$, produces an antinociceptive effect, and is involved in modulating depression, anxiety, blood pressure, etc. Remarkably, the investigation regarding the physiological and pathological functions of spexin is still at the beginning, and the information about the molecular mechanism of the spexin is deficient. Therefore, the receptor-activated signal transduction pathways of spexin and clinical trials are needed to be explored in the

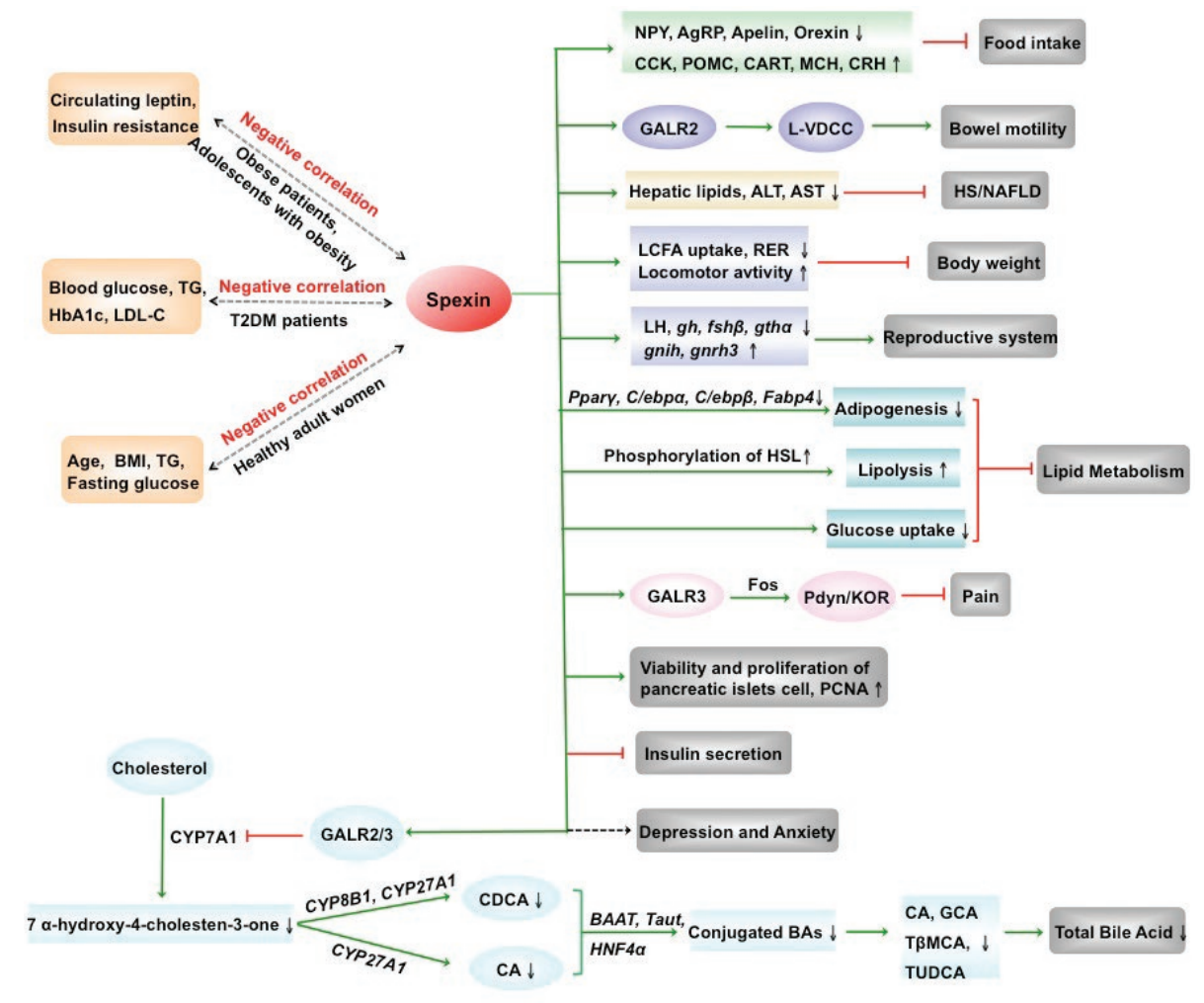

FIGURE 2 | The physiological and pathophysiological effects of spexin. AgRP, agouti gene-related protein; ALT, alanine transaminase; AST, aspartate aminotransferase; BAAT, bile acid CoA: amino acid N-acyltransferase; BAs, bile acids; BMI, body mass index; CA, cholic acid; CART, cocaine-amphetamine regulated transcript; CCK, cholecystokinin; CDCA, chenodeoxycholate; $\mathrm{CRH}$, corticotropin-releasing hormone; CYP27A1, sterol 27-hydroxylase; CYP7A1, cholesterol $7 \alpha$-hydroxylase 1; CYP8B1, sterol 12 $\alpha$-hydroxylase; fsh $\beta$, follicle-stimulating hormone $\beta$; GalR2, galanin receptor 2; GalR3, galanin receptor 3; GCA, glycocholate; gh, growth hormone; gnih, gonadotropin inhibitory hormone; gnrh3, gonadotropin-releasing hormone 3; gth $\alpha$, gonadotropin hormone $\alpha$; HbA1c, hemoglobin A1c; HNF4a, hepatocyte nuclear factor 4 alpha; HS/NAFLD, hepatic steatosis/nonalcoholic fatty liver disease; KOR, k-opioid receptor; LCFA, long-chain fatty acid; LDL-C, low-density lipoprotein-cholesterol; LH, luteinizing hormone; L-VDCC, L-type voltage-dependent calcium channel; $\mathrm{MCH}$, melanin-concentrating hormone; NPY, neuropeptides Y; PCNA, proliferating cell nuclear antigen; Pdyn, prodynorphin; POMC, proopiomelanocortin; RER, respiratory exchange ratio; T2DM, type 2 diabetes mellitus; T $\beta$-MCA, tauro- $\beta$-muricholate; taut, taurine transporter gene; TG, triglyceride; TUDCA, tauroursodeoxycholate; $\uparrow$, increase; $\downarrow$, decrease; green arrow, promotion; red arrow, inhibition. (Partial figure originated from Lin et al., 2018a,b). 
future. Moreover, whether spexin could across the blood-brain barrier is still unknown. Development of the more stable and not easily degradable spexin analogues might provide us a new therapeutic tool for liver disease in the future.

\section{AUTHOR CONTRIBUTIONS}

S-YL, Y-CZ, and X-MZ wrote the manuscript. S-YL prepared the figures. $\mathrm{W}-\mathrm{DC}$ and $\mathrm{Y}-\mathrm{DW}$ edited and revised the manuscript.

\section{FUNDING}

This work was supported by the National Natural Science Foundation of China (Grant No. 81600974) to S-YL; the National Natural Science Foundation of China (Grant No. 81472232) to W-DC; the National Natural Science Foundation of China (Grant Nos. 81672433 and 81370537) to Y-DW; Program for Science \& Technology Innovation Talents in Universities of

\section{REFERENCES}

Al-Daghri, N. M., Alenad, A., Al-Hazmi, H., Amer, O. E., Hussain, S. D., and Alokail, M. S. (2018b). Spexin levels are associated with metabolic syndrome components. Dis. Markers 2018:1679690. doi: 10.1155/2018/1679690

Al-Daghri, N. M., Al-Hazmi, H. A., Al-Ajlan, A., Masoud, M. S., Al-Amro, A., Al-Ghamdi, A., et al. (2018a). Associations of spexin and cardiometabolic parameters among women with and without gestational diabetes mellitus. Saudi J. Biol. Sci. 25, 710-714. doi: 10.1016/j.sjbs.2018.01.002

Al-Daghri, N. M., Sabico, S., Al-Hazmi, H., Alenad, A. M., Al-Amro, A., Al-Ghamdi, A., et al. (2019). Circulating spexin levels are influenced by the presence or absence of gestational diabetes. Cytokine 113, 291-295. doi: 10.1016/j.cyto.2018.07.023

Chen, T., Wang, F., Chu, Z., Sun, L., Lv, H., Zhou, W., et al. (2019). Circulating spexin decreased and negatively correlated with systemic insulin sensitivity and pancreatic beta cell function in obese children. Ann. Nutr. Metab. 74, 125-131. doi: 10.1159/000496459

Deng, S.-P., Chen, H.-P., Zhai, Y., Jia, L.-Y., Liu, J.-Y., Wang, M., et al. (2018). Molecular cloning, characterization and expression analysis of spexin in spotted scat (Scatophagus argus). Gen. Comp. Endocrinol. 266, 60-66. doi: 10.1016/j.ygcen.2018.04.018

Gu, L., Gu, M., Zhang, Y., Yan, S., Li, N., Ma, Y., et al. (2016). Correlation between serum spexin level and blood glucose and lipids in patients with type 2 diabetes. Shanghai Med. J. 39, 142-145. (in Chinese).

Gu, L., Ma, Y., Gu, M., Zhang, Y., Yan, S., Li, N., et al. (2015). Spexin peptide is expressed in human endocrine and epithelial tissues and reduced after glucose load in type 2 diabetes. Peptides 71, 232-239. doi: 10.1016/j. peptides.2015.07.018

Hodges, S. K., Teague, A. M., Dasari, P. S., and Short, K. R. (2018). Effect of obesity and type 2 diabetes, and glucose ingestion on circulating spexin concentration in adolescents. Pediatr. Diabetes 19, 212-216. doi: 10.1111/pedi.12549

Jasmine, F. G., Walewski, J. L., Anglade, D., and Berk, P. (2016). Regulation of hepatocellular fatty acid uptake in mouse models of fatty liver disease with and without functional leptin signaling: roles of $\mathrm{nfkb}$ and srebp- $1 \mathrm{c}$ and the effects of spexin. Semin. Liver Dis. 36, 360-372. doi: 10.1055/s-0036-1597248

Karaca, A., Bakar-Ates, F., and Ersoz Gulcelik, N. (2018). Decreased spexin levels in patients with type 1 and type 2 diabetes. Med. Princ. Pract. 27, 549-554. doi: 10.1159/000493482

Kim, D.-K., Yun, S., Son, G. H., Hwang, J.-I., Park, C. R., Kim, J. I., et al. (2014). Coevolution of the spexin/galanin/kisspeptin family: spexin activates galanin receptor type II and III. Endocrinology 155, 1864-1873. doi: 10.1210/ en.2013-2106
Henan Province (HASTIT, Grant No. 13HASTIT024) and Plan for Scientific Innovation Talent of Henan Province to W-DC; and the Key Science and Technology Program of Henan Province in China (Grant No. 192102310080), the Key Scientific Research Program for Universities of Henan Province in China (Grant No. 17A310003), the Fundamental Research Funds of Henan University (Grant No. yqpy20170040), the Key Science and Technology Program of Kaifeng City in China (Grant Nos. 1803034 and 1903019), and the Scientific Research Foundation of Henan University (Grant No. 2015YBZR050) to S-YL; and the Fundamental Research Funds for the Central Universities and Research projects on biomedical transformation of ChinaJapan Friendship Hospital (Grant No. PYBZ1803) to Y-DW.

\section{ACKNOWLEDGMENTS}

We would like to thank the other members of our group for their suggestions and help in this article. We apologize to colleagues whose work could not be cited due to space limitations.

Kolodziejski, P. A., Pruszynska-Oszmalek, E., Micker, M., Skrzypski, M., Wojciechowicz, T., Szwarckopf, P., et al. (2018). Spexin: a novel regulator of adipogenesis and fat tissue metabolism. Biochim. Biophys. Acta Mol. Cell Biol. Lipids 1863, 1228-1236. doi: 10.1016/j.bbalip.2018.08.001

Kumar, S., Hossain, M. J., Javed, A., Kullo, I. J., and Balagopal, P. B. (2017). Relationship of circulating spexin with markers of cardiovascular disease: a pilot study in adolescents with obesity. Pediatr. Obes. 13, 374-380. doi: 10.1111/ijpo.12249

Kumar, S., Hossain, J., Nader, N., Aguirre, R., Sriram, S., and Balagopal, P. B. (2016). Decreased circulating levels of spexin in obese children. J. Clin. Endocrinol. Metab. 101, 2931-2936. doi: 10.1210/jc.2016-1177

Lang, R., Gundlach, A. L., Holmes, F. E., Hobson, S. A., Wynick, D., Hoekfelt, T., et al. (2015). Physiology, signaling, and pharmacology of galanin peptides and receptors: three decades of emerging diversity. Pharmacol. Rev. 67, 118-175. doi: 10.1124/pr.112.006536

Li, S., Liu, Q., Xiao, L., Chen, H., Li, G., Zhang, Y., et al. (2016). Molecular cloning and functional characterization of spexin in orange-spotted grouper (Epinephelus coioides). Comp. Biochem. Physiol. B Biochem. Mol. Biol. 196, 85-91. doi: 10.1016/j.cbpb.2016.02.009

Lin, C.-Y., Huang, T., Zhao, L., Zhong, L. L. D., Lam, W. C., Fan, B.-M., et al. (2018a). Circulating Spexin levels negatively correlate with age, BMI, fasting glucose, and triglycerides in healthy adult women. J. Endocr. Soc. 2, 409-419. doi: 10.1210/js.2018-00020

Lin, C.-y., Zhang, M., Huang, T., Yang, L.-1., Fu, H.-b., Zhao, L., et al. (2015). Spexin enhances bowel movement through activating L-type voltage-dependent calcium channel via galanin receptor 2 in mice. Sci. Rep. 5:12095. doi: $10.1038 /$ srep 12095

Lin, C.-y., Zhao, L., Huang, T., Lu, L., Khan, M., Liu, J., et al. (2018b). Spexin acts as novel regulator for bile acid synthesis. Front. Physiol. 9:378. doi: 10.3389/fphys.2018.00378

Liu, Y., Li, S., Qi, X., Zhou, W., Liu, X., Lin, H., et al. (2013). A novel neuropeptide in suppressing luteinizing hormone release in goldfish, Carassius auratus. Mol. Cell. Endocrinol. 374, 65-72. doi: 10.1016/j.mce.2013.04.008

Lv, S.-Y., Cui, B., Yang, Y., Du, H., Zhang, X., Zhou, Y., et al. (2019). Spexin/ NPQ induces fos and produces antinociceptive effect against inflammatory pain in the mouse model. Am. J. Pathol. 189, 886-899. doi: 10.1016/j.ajpath.2018.12.009

Ma, A., Bai, J., He, M., and Wong, A. O. L. (2018). Spexin as a neuroendocrine signal with emerging functions. Gen. Comp. Endocrinol. 265, 90-96. doi: 10.1016/j.ygcen.2018.01.015

Ma, A., He, M., Bai, J., Wong, M. K. H., Ko, W. K. W., and Wong, A. O. L. (2017). Dual role of insulin in spexin regulation: functional link between food intake and spexin expression in a fish model. Endocrinology 158, 560-577. doi: 10.1210/en.2016-1534 
Mirabeau, O., Severini, C., Audero, E., Gascuel, O., Possenti, R., Birney, E., et al. (2007). Identification of novel peptide hormones in the human proteome by hidden Markov model screening. Genome Res. 17, 320-327. doi: 10.1101/ gr.5755407

Moazen, P., Taherianfard, M., Ahmadi Soleimani, M., and Norozpor, M. (2017). Synergistic effect of spexin and progesterone on pain sensitivity attenuation in ovariectomized rats. Clin. Exp. Pharmacol. Physiol. 45, 349-354. doi: 10.1111/1440-1681.12862

Palasz, A., Palka, M., Filipczyk, L., Menezes, I. C., Rojczyk, E., Worthington, J. J., et al. (2018). Effect of long-term treatment with classical neuroleptics on $\mathrm{NPQ} /$ spexin, kisspeptin and POMC mRNA expression in the male rat amygdala. J. Neural. Transm.(Vienna). 125, 1099-1105. doi: 10.1007/s00702-018-1868-2

Palasz, A., Suszka-Switek, A., Filipczyk, L., Bogus, K., Rojczyk, E., Worthington, J., et al. (2016). Escitalopram affects spexin expression in the rat hypothalamus, hippocampus and striatum. Pharmacol. Rep. 68, 1326-1331. doi: 10.1016/j. pharep.2016.09.002

Petrescu, O., Fan, X., Gentileschi, P., Hossain, S., Bradbury, M., Gagner, M., et al. (2005). Long-chain fatty acid uptake is upregulated in omental adipocytes from patients undergoing bariatric surgery for obesity. Int. J. Obes.(Lond). 29, 196-203. doi: 10.1038/sj.ijo.0802868

Pirzeh, L., and Taherianfard, M. (2014). Effect of intra hippocampal CA1 injection of spexin on pain sensitivity in female rat. Bull Env. Pharmacol. Life Sci. 3, 71-74.

Porzionato, A., Rucinski, M., Macchi, V., Stecco, C., Malendowicz, L. K., and De Caro, R. (2010). Spexin expression in normal rat tissues. J. Histochem. Cytochem. 58, 825-837. doi: 10.1369/jhc.2010.956300

Porzionato, A., Rucinski, M., Macchi, V., Stecco, C., Sarasin, G., Sfriso, M. M., et al. (2012). Spexin is expressed in the carotid body and is upregulated by postnatal hyperoxia exposure. Adv. Exp. Med. Biol. 758, 207-213. doi: 10.1007/978-94-007-4584-1_29

Reyes-Alcaraz, A., Lee, Y.-N., Son, G. H., Kim, N. H., Kim, D.-K., Yun, S., et al. (2016). Development of spexin-based human galanin receptor type II-specific agonists with increased stability in serum and anxiolytic effect in mice. Sci. Rep. 6:21453. doi: 10.1038/srep21453

Reyes-Alcaraz, A., Lee, Y.-N., Yun, S., Hwang, J.-I., and Seong, J. Y. (2018). Conformational signatures in beta-arrestin2 reveal natural biased agonism at a G-protein-coupled receptor. Commun. Biol. 1, 128-128. doi: 10.1038/ s42003-018-0134-3

Rucinski, M., Porzionato, A., Ziolkowska, A., Szyszka, M., Macchi, V., De Caro, R., et al. (2010). Expression of the spexin gene in the rat adrenal gland and evidences suggesting that spexin inhibits adrenocortical cell proliferation. Peptides 31, 676-682. doi: 10.1016/j.peptides.2009.12.025

Sassek, M., Kolodziejski, P. A., Strowski, M. Z., Nogowski, L., Nowak, K. W., and Mackowiak, P. (2018a). Spexin modulates functions of rat endocrine pancreatic cells. Pancreas 47, 904-909. doi: 10.1097/mpa.0000000000001083

Sassek, M., Kolodziejski, P. A., Szczepankiewicz, D., and Pruszynska-Oszmalek, E. (2018b). Spexin in the physiology of pancreatic islets-mutual interactions with insulin. Endocrine 63, 513-519. doi: 10.1007/s12020-018-1766-2
Sonmez, K., Zaveri, N. T., Kerman, I. A., Burke, S., Neal, C. R., Xie, X., et al. (2009). Evolutionary sequence modeling for discovery of peptide hormones. PLoS Comput. Biol. 5:e1000258. doi: 10.1371/journal.pcbi.1000258

Toll, L., Khroyan, T. V., Sonmez, K., Ozawa, A., Lindberg, I., McLaughlin, J. P., et al. (2012). Peptides derived from the prohormone proNPQ/spexin are potent central modulators of cardiovascular and renal function and nociception. FASEB J. 26, 947-954. doi: 10.1096/fj.11-192831

Trudeau, V. L. (2018). Facing the challenges of neuropeptide gene knockouts: why do they not inhibit reproduction in adult teleost fish? Front. Neurosci. 12:302. doi: $10.3389 /$ fnins.2018.00302

Walewski, J. L., and Berk, P. D. (2013). Obesity-related genes and their proteins and uses thereof (Google Patents). doi: US20130274181 A1

Walewski, J. L., Ge, F., Lobdell, H., Levin, N., Schwartz, G. J., Vasselli, J. R., et al. (2014). Spexin is a novel human peptide that reduces adipocyte uptake of long chain fatty acids and causes weight loss in rodents with diet-induced obesity. Obesity 22, 1643-1652. doi: 10.1002/oby.20725

Wan, B., Wang, X.-R., Zhou, Y.-B., Zhang, X., Huo, K., and Han, Z.-G. (2010). C120RF39, a novel secreted protein with a typical amidation processing signal. Biosci. Rep. 30, 1-10. doi: 10.1042/bsr20080156

Wang, S., Wang, B., and Chen, S. (2018). Spexin in the half-smooth tongue sole (Cynoglossus semilaevis): molecular cloning, expression profiles, and physiological effects. Fish Physiol. Biochem. 44, 829-839. doi: 10.1007/ s10695-018-0472-6

Wang, X., Wang, S., Feng, W., Wang, R., Zhang, H., Yang, X., et al. (2016). "Research progress of Spexin in energy metabolism" in Chemistry of Life, vol. 36, 852-855. (in Chinese).

Wong, M. K., Sze, K. H., Chen, T., Cho, C. K., Law, H. C., Chu, I. K., et al. (2013). Goldfish spexin: solution structure and novel function as a satiety factor in feeding control. Am. J. Physiol. Endocrinol. Metab. 305, E348-E366. doi: 10.1152/ajpendo.00141.2013

Wu, H., Lin, F., Chen, H., Liu, J., Gao, Y., Zhang, X., et al. (2015). Ya-fish (Schizothorax prenanti) spexin: identification, tissue distribution and mRNA expression responses to periprandial and fasting. Fish Physiol. Biochem. 42, 39-49. doi: 10.1007/s10695-015-0115-0

Zheng, B., Li, S., Liu, Y., Li, Y., Chen, H., Tang, H., et al. (2017). Spexin suppress food intake in Zebrafish: evidence from gene knockout study. Sci. Rep. 7:14643. doi: 10.1038/s41598-017-15138-6

Conflict of Interest Statement: The authors declare that the research was conducted in the absence of any commercial or financial relationships that could be construed as a potential conflict of interest.

Copyright (c) 2019 Lv, Zhou, Zhang, Chen and Wang. This is an open-access article distributed under the terms of the Creative Commons Attribution License (CC BY). The use, distribution or reproduction in other forums is permitted, provided the original author(s) and the copyright owner(s) are credited and that the original publication in this journal is cited, in accordance with accepted academic practice. No use, distribution or reproduction is permitted which does not comply with these terms. 